\title{
A comparison of structural connectivity in anxious depression versus non-anxious depression
}

\author{
Lauren Delaparte a, b, *, Fang-Cheng Yeh ${ }^{\mathrm{c}}$, Phil Adams ${ }^{\mathrm{e}}$, Ashley Malchow ${ }^{\mathrm{h}}$, \\ Madhukar H. Trivedi ${ }^{\text {h }}$, Maria A. Oquendo ${ }^{e}$, Thilo Deckersbach ${ }^{f}$, Todd Ogden ${ }^{e}$, \\ Diego A. Pizzagalli ${ }^{i}$, Maurizio Fava ${ }^{\mathrm{f}}$, Crystal Cooper ${ }^{\mathrm{h}}$, Melvin McInnis ${ }^{\mathrm{g}}$, Benji T. Kurian ${ }^{\mathrm{h}}$, \\ Myrna M. Weissman ${ }^{\text {e, }}$ Patrick J. McGrath ${ }^{\mathrm{e}}$, Daniel N. Klein ${ }^{\mathrm{a}}$, Ramin V. Parsey ${ }^{\mathrm{b}, \mathrm{d}}$, \\ Christine DeLorenzo ${ }^{\text {b }}$
}

a Department of Psychology, Stony Brook University, Stony Brook, NY, USA

${ }^{\mathrm{b}}$ Department of Psychiatry, Stony Brook University, Stony Brook, NY, USA

${ }^{c}$ Department of Neurological Surgery, University of Pittsburgh, Pittsburgh, USA

d Department of Radiology, Stony Brook University, Stony Brook, NY, USA

e Department of Psychiatry, New York State Psychiatric Institute, Columbia University College of Physicians and Surgeons, New York, NY, USA

${ }^{\mathrm{f}}$ Department of Psychiatry, Massachusetts General Hospital, Boston, MA, USA

$\mathrm{g}^{\mathrm{D}}$ Department of Psychiatry, University of Michigan School of Medicine, Ann Arbor, MI, USA

${ }^{\mathrm{h}}$ Department of Psychiatry, University of Texas Southwestern Medical Center, Dallas, TX, USA

${ }^{\mathrm{i}}$ Department of Psychiatry, Harvard Medical School - McLean Hospital, USA

\section{A R T I C L E I N F O}

\section{Article history:}

Received 9 September 2016

Received in revised form

16 December 2016

Accepted 19 January 2017

\section{Keywords:}

Diffusion tensor imaging

Anxious-depression

Anxiety

Depression

White matter health

Fractional anisotropy

\begin{abstract}
A B S T R A C T
Background: Major depressive disorder (MDD) and anxiety disorders are highly co-morbid. Research has shown conflicting evidence for white matter alteration and amygdala volume reduction in mood and anxiety disorders. To date, no studies have examined differences in structural connectivity between anxious depressed and non-anxious depressed individuals. This study compared fractional anisotropy (FA) and density of selected white matter tracts and amygdala volume between anxious depressed and non-anxious depressed individuals.

Methods: 64- direction DTI and T1 scans were collected from 110 unmedicated subjects with MDD, 39 of whom had a co-morbid anxiety disorder diagnosis. Region of interest (ROI) and tractography methods were performed to calculate amygdala volume and FA in the uncinate fasciculus, respectively. Diffusion connectometry was performed to identify whole brain group differences in white matter health. Correlations were computed between biological and clinical measures.

Results: Tractography and ROI analyses showed no significant differences between bilateral FA values or bilateral amygdala volumes when comparing the anxious depressed and non-anxious depressed groups. The diffusion connectometry analysis showed no significant differences in anisotropy between the groups. Furthermore, there were no significant relationships between MRI-based and clinical measures. Conclusion: The lack of group differences could indicate that structural connectivity and amygdalae volumes of those with anxious-depression are not significantly altered by a co-morbid anxiety disorder. Improving understanding of anxiety co-morbid with MDD would facilitate development of treatments that more accurately target the underlying networks.
\end{abstract}

() 2017 Elsevier Ltd. All rights reserved.
* Corresponding author. Department of Psychology, Stony Brook University, Stony Brook, NY, 11794-8101, USA.

E-mail address: Lauren.Delaparte@stonybrook.edu (L. Delaparte).

\section{Introduction}

Anxiety and depression are highly co-morbid (Fava et al., 2000). The Diagnostic and Statistical Manual of Mental Disorders (DSM-5; American Psychiatric Association, 2000) recently recognized this with a novel specifier termed "anxious distress," while the 
International Classification of Disease (ICD; World Health Organization, 1992) initially acknowledged anxious depression with the concept of "mixed anxiety-depression." However, evidence of highly overlapping genetic influences raises questions of whether anxiety disorders and major depressive disorder are distinct conditions (Kendler et al., 1992), and there is mixed evidence for a biological basis of the DSM modifier.

The DSM's definition of anxious depression requires a diagnosis of a depressive disorder with anxious features, like "constant worry." In contrast, the ICD diagnosis denotes sub-threshold symptoms of both anxiety and depression. In addition, past studies have defined anxious depression in a number of other ways, such as a DSM IV-TR diagnosis of major depressive disorder (MDD) with a score greater than or equal to seven on the composite of somatization and psychic anxiety items on the Hamilton Depression Rating Scale (Fava et al., 2008) and as meeting criteria for both MDD and an anxiety disorder.

A better understanding of the underlying biology could help define anxious-depression and address the current lack of consensus. Most research to date that explores potential biomarkers for mood and anxiety disorders has focused on the neural correlates of anxiety disorders and depression, without controlling for co-morbidities (e.g., Cullen et al., 2010; Steffens et al., 2011; Strawn et al., 2012); little research has been conducted on neural correlates and potential biomarkers for anxious depression. However, given that comorbidity is more common than not in depression (Avenevoli et al., 2015), it is important to explore potential biomarkers of these disorders as they actually exist in the population, with consideration for nuanced comorbid symptoms. Accordingly, a thorough understanding of the underlying biology of these co-morbid disorders could aid considerably with diagnosis and treatment assignments of clinical populations.

Here we used diffusion MRI, diffusion tensor imaging (DTI), to study how brain connectivity differs between anxious depressed and non-anxious depressed individuals. DTI is an MRI modality that uses a pulse sequence to quantify diffusion of water molecules in the brain and infer macroscopic tissue properties (e.g., white matter integrity). Several diffusion MRI analysis methods have been proposed to examine diffusion signals. In this study, we used both conventional and novel approaches (Abhinav et al., 2014) to examine the imaging data. The conventional approach involved using a tensor model (Basser et al., 1994) to provide the outcome measure: fractional anisotropy (FA). These values are proxies for strength of directionality of water diffusion along different axes. Specifically, fractional anisotropy measures the ratio of diffusivity between the primary and radial axis of a fiber bundle. Previous studies have used DTI sequences with fewer directions, which represents an important limitation owing to less accurate accounts of the strength of diffusion along multiple axes (Giannelli et al., 2010). Conventional analysis also included tractography (Abhinav et al., 2014; Jbabdi et al., 2015), which utilizes fiber tracking to map connections between regions of interest in the brain. However, the accuracy of tractography has been questioned (Reveley et al., 2015) and its limitations may render track-specific analysis inconclusive. Recently, a new method called connectometry was proposed to bypass the limitation of fiber tracking by more accurately reflecting the structure and density of the white matter tracts while accounting for crossing fibers and partial volume effects (Yeh et al., 2016).

The amygdala plays a significant role in fear learning and anxiety (LeDoux, 2003). Functional MRI studies have shown hyperactivation of the amygdala in most anxiety disorders, namely social anxiety disorder, generalized anxiety disorder, specific phobia and panic disorder (Shin and Liberzon, 2010; Linares et al., 2012). Structural studies have described decreased left amygdalar volume in sub-clinically anxious individuals (Blackmon et al., 2011). As far as we know, there is no existing work on distinctions in white matter abnormalities for the various anxiety disorders. In particular, previous research has shown that the uncinate fasciculus, the white matter tract that connects the amygdala to the orbitofrontal cortex, is altered in generalized anxiety disorder (Tromp et al., 2012; Hettema et al., 2012), and social anxiety disorder (Phan et al., 2009). Diminished connectivity of the uncinate fasciculus, specifically from the orbitofrontal cortex to the amygdala, may

Table 1

Demographic and clinical data for each group.

\begin{tabular}{|c|c|c|c|}
\hline \multicolumn{4}{|l|}{ Descriptive statistics } \\
\hline Demographic variables & Anxious depressed $(\mathrm{N}=39)$ & Non-anxious MDD $(\mathrm{N}=71)$ & $P$ values \\
\hline Sex, N (\%) Male & $17(44 \%)$ & $23(32 \%)$ & \\
\hline Age, years & $35.52 \pm 2.37$ & $34.33 \pm 1.68$ & 0.95 \\
\hline \multicolumn{4}{|l|}{ Clinical Variables } \\
\hline HAM-D 24-item & $20.61 \pm 4.89$ & $20.41 \pm 4.42$ & 0.59 \\
\hline HAM Anxiety Factor & $5.11 \pm 1.74$ & $5.19 \pm 1.79$ & 0.45 \\
\hline Age of Onset & $17.03 \pm 6.85$ & $15.24 \pm 5.02$ & 0.19 \\
\hline Length Current MDE & $42.14 \pm 67.05$ & $32.79 \pm 57.56$ & 0.49 \\
\hline \multicolumn{4}{|l|}{ Diagnoses } \\
\hline Social Phobia & $14(36 \%)$ & - & \\
\hline Panic Disorder & $7(18 \%)$ & - & \\
\hline Generalized Anxiety Disorder & $6(15 \%)$ & - & \\
\hline Anxiety NOS & $4(10 \%)$ & - & \\
\hline Specific Phobia & $8(21 \%)$ & - & \\
\hline
\end{tabular}

Table 2

Means, standard deviations and p values for each of the four dependent variables in the two groups.

\begin{tabular}{|c|c|c|c|}
\hline \multicolumn{4}{|l|}{ Descriptive statistics } \\
\hline Outcome measure $M(S D)$ & Anxious depressed & Non-anxious MDD & F test $\mathrm{p}$ values \\
\hline Left UF FA & $0.35(0.04)$ & $0.34(0.05)$ & 0.89 \\
\hline Right UF FA & $0.37(0.05)$ & $0.36(0.04)$ & 0.94 \\
\hline Left Amygdala Volumes $\left(\mathrm{mm}^{3}\right)$ & $1603.34(205.13)$ & $1546.20(264.34)$ & 0.52 \\
\hline Right Amygdala Volumes $\left(\mathrm{mm}^{3}\right)$ & $1727.49(239.19)$ & $1663.01(245)$ & 0.15 \\
\hline
\end{tabular}


Right Amygdala Volume and HAM-D

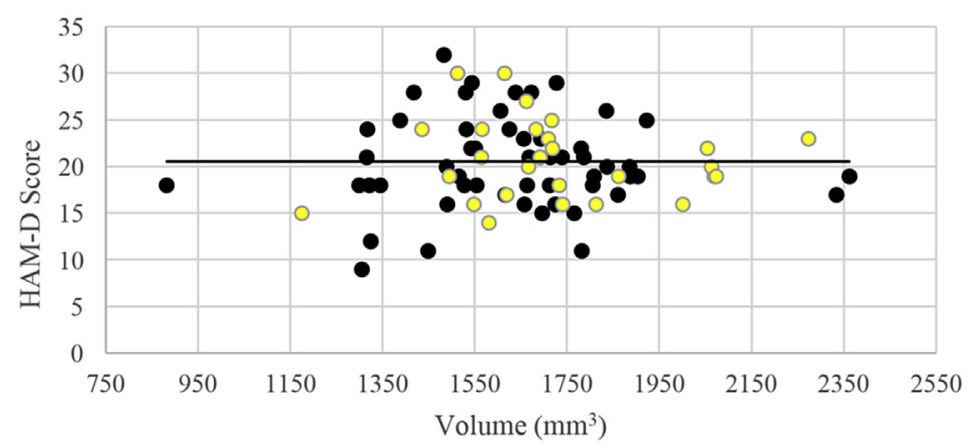

- Non-anxious depression

- Anxious depression

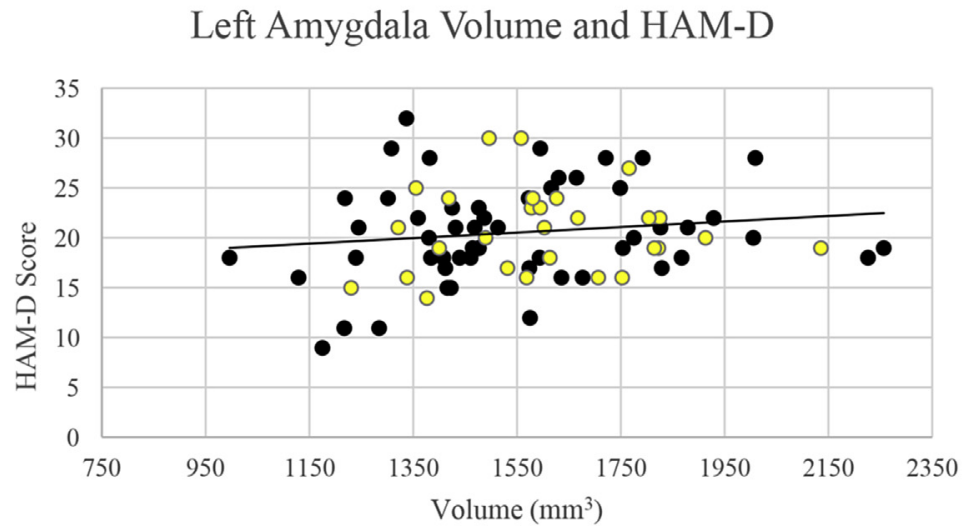

- Non-anxious depression

- Anxious depression

Fig. 1. Scatterplots of Hamilton Depression Rating Scale scores and volumes of the right $(r=-0.026, p=0.819)$ and left $(r=0.157$, $p=0.167)$ amygdalae in mm ${ }^{3}$.

reflect a reduction of top-down control leading to hyperactivation of the amygdala (Cullen, et al., 2010).

In depression, the evidence for alterations in white matter connectivity is mixed. Some studies show decreased fractional anisotropy in a number of brain regions including tracts extending from the anterior cingulate cortex to the amygdala and the uncinate fasciculus (Cullen, et al., 2010; Taylor et al., 2007), while our previous study reported no difference in tracts extending from the medialorbitofrontal cortex (Olvet et al., 2016). The mixed findings observed in the structural connectivity depression literature may be caused by failing to account for anxiety co-morbidities.

Given that they are likely to be associated based on previous knowledge of cortico-limbic neurocircuitry, this paper sought to combine the structural connectivity and volume literature by examining tracts and amygdalar volumes implicated in anxiety disorders and mood disorders. Due to converging evidence of alterations in white matter connectivity in individuals with anxiety and previous literature supporting the uncinate fasciculi as a ROI (region of interest), we hypothesize that individuals with anxious depression will exhibit reduced white matter health in the uncinate fasciculi and in other pathways when compared to nonanxious depressed individuals. We also expect that amygdala volume will be significantly decreased in those with anxious depression versus those with non-anxious depression. This is the first study to date that uses 64-direction DTI methods and diffusion connectometry to measure structural connectivity differences between anxious depressed and non-anxious depressed individuals.

\section{Methods}

\subsection{Subjects}

The Establishing Moderators/Mediators for a Biosignature of Antidepressant Response in Clinical Care (EMBARC) project enrolled participants at four different sites: Columbia University Medical Center (CU), Massachusetts General Hospital (MG), University of Michigan (UM), and University of Texas Southwestern Medical Center (TX). The Institutional Review Board for each site approved the protocol, and participants provided written informed consent.

To determine eligibility for the EMBARC protocol, subjects received a structured diagnostic interview (Structured Clinical Interview for DSM-IV Patient Version (SCID-IV-I/P; First et al., 1995), review of medical history, chart review, physical examination, routine blood tests, pregnancy test and urine toxicology test. 139 participants between the ages of 18 and 65 who met criteria for a major depressive episode (MDE) according to the DSM IV-TR enrolled in the study. Participants had to score a minimum of 14 on the Quick Inventory of Depressive Symptoms (QIDS-SR; Rush et al., 2003), as well as meet DSM-IV-TR criteria for a current MDE with an onset before 30 years of age. Exclusion criteria were a lifetime history of psychosis, bipolar disorder, or current drug or alcohol abuse (within 2 months)/dependence (within 6 months). Diagnoses of post-traumatic stress disorder and obsessive compulsive disorder were also excluded on the basis of their reclassification in the DSM-5 due to converging evidence suggesting differential circuitry, symptomatology and etiology from anxiety disorders (American Psychiatric Association, 2013; Stein et al., 

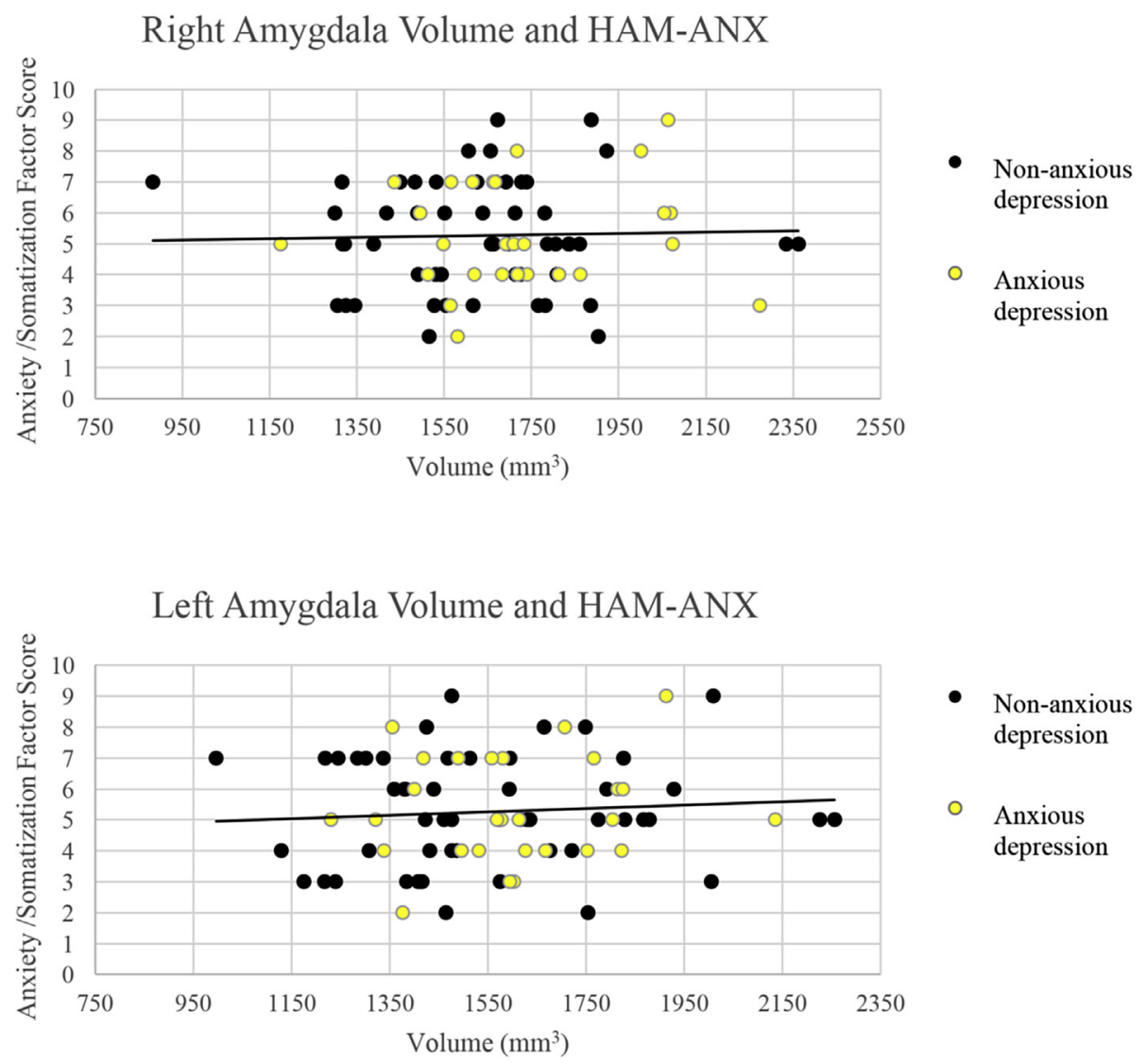

Fig. 2. Scatterplots of Hamilton-derived Anxiety/Somatization factor scores and volumes of the right $(r=0.044, p=0.704)$ and left $(r=0.102$, $p=0.373)$ amygdalae in mm ${ }^{3}$.

2010). Subjects on psychoactive medications underwent a threeweek medication washout prior to undergoing the brain scan portion of the project. Anxious depression was defined as having a primary DSM-IV diagnosis of major depressive disorder and a comorbid anxiety disorder based on the SCID-IV-I/P. Group membership was based on a clinical diagnostic interview to capture a wider range of anxiety symptoms and to form a comprehensive diagnosis. Clinical raters at each study site were trained and certified by the EMBARC National Coordinating Center.

The total sample was 139, however, 29 subjects were not included due to significant artifact in the diffusion-weighted images including head motion and poor segmentation $(\mathrm{N}=12$ anxious depressed and $\mathrm{N}=17$ non-anxious depressed). The final sample sizes were $\mathrm{N}=71$ for the non-anxious depressed group and $\mathrm{N}=39$ for the anxious depressed group. There were the following co-morbid anxiety disorder diagnoses in the group: specific phobia $(\mathrm{N}=8)$, panic disorder $(\mathrm{N}=7)$, social phobia $(\mathrm{N}=14)$, generalized anxiety disorder $(\mathrm{N}=6)$, anxiety disorder, not otherwise specified $(\mathrm{N}=4)$. All 110 depressed subjects were examined in a previous analysis (Olvet, et al., 2016); that study looked at the effects of depression diagnosis in different a priori brain regions. A more comprehensive EMBARC project methodology is described elsewhere (Trivedi, McGrath, Fava, et al., in press).

\subsection{Clinical measures}

The 24-item Hamilton Depression Rating Scale (HAM-D; Hamilton, 1960) assessed clinician-rated depression severity. The anxiety/somatization factor score derived from 6 items on the HAMD (Fava et al., 2004) was used as a dimensional anxiety measure.

\subsection{Image acquisition}

All participants underwent MRI scanning in one of the following scanners: Columbia University Medical Center (GE Signa HDx), Massachusetts General Hospital (Siemens TrioTim), University of Michigan (Philips Ingenia) and University of Texas Southwestern Medical Center (Philips Achieva). Prior to the project's initiation, physicists at each site worked to streamline the data acquisition and parameters and conducted phantom studies, which increase reproducibility of findings (Zhu et al., 2011). While we took great care to minimize inter-site acquisition effects, we recognize that eliminating these differences is impossible and included site as a covariate. Further, certain parameters must be optimized according to each site's scanner; perfect uniformity in parameters across different scanners, if possible, could produce a disparity in image quality, and not necessarily improve inter-site compatibility. Further, we tried to maximize uniformity by performing all DTI analysis at a single site. Since our goal is widespread clinical applicability, it was necessary to include multiple sites, enforcing uniformity to as great an extent as possible. Cortical volume was estimated by Freesurfer (http://surfer.nmr.mgh.harvard.edu/). In a previous study performed by our group, Freesurfer-derived volumes were reliable in a test-retest analysis across the four sites with intraclass correlation coefficients (ICC) and percent differences (PD) of 0.88 and 1.39, respectively (Iscan et al., 2015). Although that study does not report amygdala extraction reliability, our group implemented the same pipeline for the current study and manually inspected all outlined regions of interest. In a test-retest analysis of amygdala volume in the same sample, across the four sites, the average percent difference estimate was $7 \%$. At all sites, 
Right Weighted FA and HAM-D

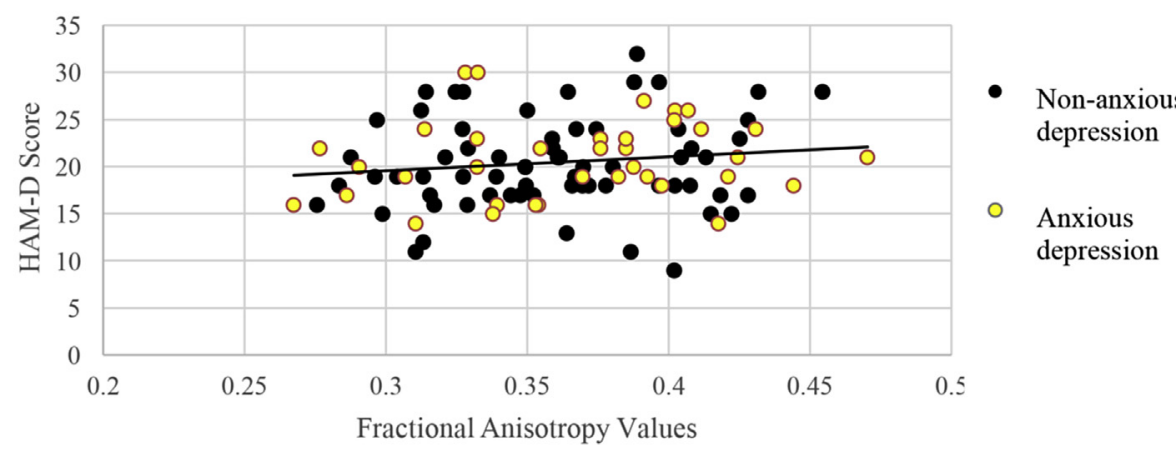

Left Weighted FA and HAM-D

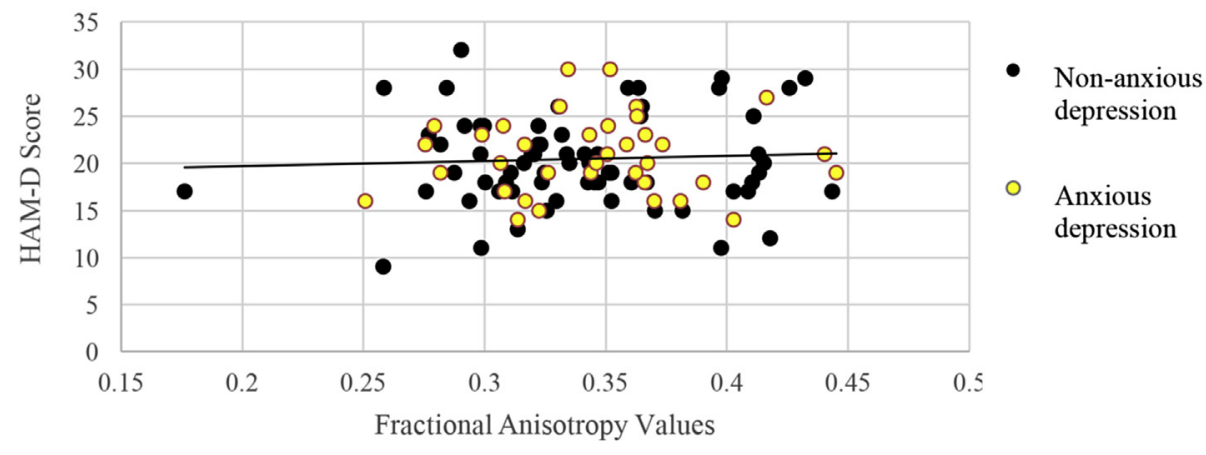

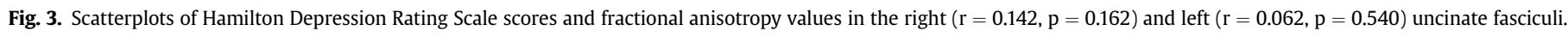

structural MRIs were acquired using the following parameters: TR (repetition time): 5.9 (Fig. 6) -8.2 ms; TE (echo time): 2.4-3.7 ms; Flip Angle: $9^{\circ}-12^{\circ}$; slice thickness: $1 \mathrm{~mm}$; FOV (field of view): $256 \mathrm{~mm} \times 256 \mathrm{~mm}$; voxel dimensions: $1 \mathrm{~mm} \times 1 \mathrm{~mm} \times 1 \mathrm{~mm}$; acquisition matrix: $240 \times 240,256 \times 243$ or $256 \times 256$; acceleration factor: 2; and 174-178 sagittal slices. Diffusion images were acquired using a single-shot EPI (echo planar imaging) sequence. Scan parameters were as follows: $\mathrm{TR}=8310-9500 \mathrm{~ms}$; $\mathrm{TE}=95-96.3 \mathrm{~ms}$; Flip Angle $90^{\circ}$; slice thickness $=2.5 \mathrm{~mm}$; FOV $=240 \times 240 \mathrm{~mm}$; voxel dimensions $2.5 \mathrm{~mm} \times 2.5 \mathrm{~mm} \times 2.5 \mathrm{~mm}$ or $1.9 \mathrm{~mm} \times 1.9 \mathrm{~mm} \times 2.5 \mathrm{~mm}$; acquisition matrix $=96 \times 96$; b value $=1000 \mathrm{~s} / \mathrm{mm}^{2}$; and 64 collinear directions with 1 or 5 non-weighted images. DTI scan time was approximately $10 \mathrm{~min}$.

\subsection{Image processing}

\subsubsection{Diffusion Tensor Imaging (DTI)}

All diffusion-weighted and T1 images were processed at Stony Brook University. Each diffusion-weighted image underwent a battery of quality assurance tests for common artifacts, including ghost, ring, slice-wise intensity, venetian blind, and gradient-wise motion artifacts (Liu et al., 2010). Using the eddy current correction routine within FSL (FMRIB Software Library, http://www.fmrib. ox.ac.uk/fsl/), diffusion-weighted images were corrected for distortion produced by gradient coils and head motion. FSL's Brain Extraction Tool (BET) was used to distinguish and remove nonbrain tissue from the image. Then, FA was estimated using Camino [http://web4.cs.ucl.ac.uk/research/medic/camino/pmwiki/ pmwiki.php], which computes the least-squares-fit diffusion tensor with non-linear optimization using a Levenburg-Marquardt algorithm, constrained to be positive by fitting its Cholesky decomposition (Alexander and Barker, 2005; Jones and Basser, 2004).

\subsubsection{Region of Interest (ROI) determinations}

Using the Bias Field Corrector in Advanced Normalization Tools (ANTS; http://www.picsl.upenn.edu/ANTS/), the T1 anatomical images were cropped and corrected for non-uniformity. The cortical parcellation tool based on the Desikan-Killiany (Desikan et al., 2006) atlas and the subcortical parcellation tool (Fischl et al., 2002) in Freesurfer were used to delineate regions of interest (ROIs) after which, they were manually inspected. White matter voxels were labeled based on the nearest cortical voxel (Salat et al., 2009). In order to position ROIs in DTI space, the DTI image was coregistered to the cropped T1 image using ANTS, and the inverse transformation was applied to the cortical map. Average FA values were computed in white matter for each ROI. To minimize comparisons, two bilateral ROIs were chosen a priori based on reported white matter deficits in the MDD, anxiety, and anxious depression literature: uncinate fasciculi and amygdala (Baur et al., 2012; Hettema, et al., 2012; Phan, et al., 2009; Tromp, et al., 2012).

\subsubsection{Tractography analysis}

Using FMRIB's Diffusion Toolbox (FDT; Behrens et al., 2007), probabilistic tractography was conducted. This algorithm repeatedly samples from the principal diffusion direction to calculate probabilistic streamlines passing through each voxel. This method generates the probability of connections from the seed (medial orbitofrontal cortex) to the target (amygdala). In running the algorithm, the tract curvature threshold was set to $0.2 \mathrm{~mm}$, the maximum number of steps per sample to 2000 , length of each step 


\section{Right Weighted FA and HAM-DANX}
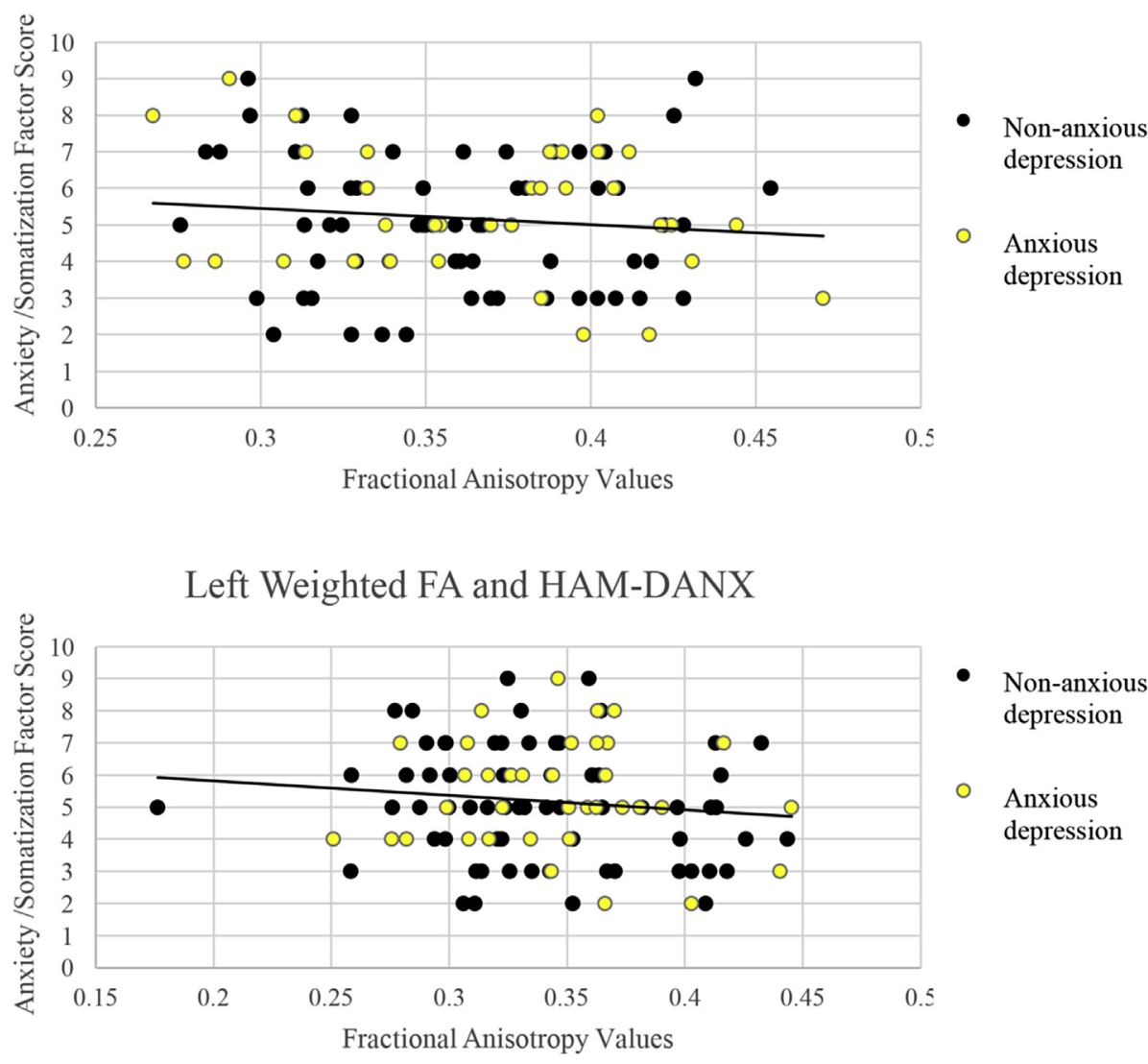

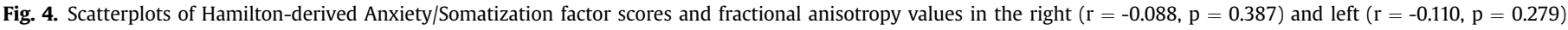
uncinate fasciculi.

to $0.5 \mathrm{~mm}$, and 5000 samples. The weighted average of FA within the defined tracts was computed by first multiplying the voxelbased FA by the probability of connection at each voxel, then dividing by the sum of probabilities.

\section{Connectometry}

We further explored differences between groups by performing connectometry (Yeh et al., 2013a) with publicly available software, DSI studio (http://dsi-studio.labsolver.org). As a complement to traditional DTI methods, diffusion connectometry measures white matter tract density by calculating spin distribution function (SDF) in every voxel in the brain and length of affected track. Using qspace diffeomorphic reconstruction (Yeh and Tseng, 2011) with a diffusion sampling length ratio of 1.25 , the diffusion data were reconstructed. The output resolution was $2 \mathrm{~mm}$. A multiple regression model was used including age, sex and site as covariates. Local fiber directions with a percentage difference greater than $5 \%$, $10 \%, 15 \%$, and $20 \%$ were connected respectively using a deterministic fiber-tracking algorithm (Yeh et al., 2013b). Tracts with connected length greater than $40 \mathrm{~mm}$ were included. The false discovery rate was estimated by applying a total of 2000 randomized permutations.

\subsection{Statistical analysis}

Comparisons between the anxious depressed group and depressed only group were evaluated statistically using $\mathrm{R}$ ( $\mathrm{R}$ Core Team, 2013). Because we were testing for differences in the means of dependent variables, we used an analysis of covariance (ANCOVA). The ANCOVA compared the variances of the four dependent variables: fractional anisotropy of the left and right uncinate fasciculi, and volume of the left and right amygdalae. The independent variable was anxious depression or non-anxious depression based on a SCID diagnosis.

In order to reduce error or noise associated with differences in the outcome measures not attributable to clinical designation, age, sex and site were included as covariates. Total brain volume was not included as a covariate because when examining volumetric differences, doing so can lead to removal of disease-related effects. However, age and sex, covariates included in the current study, are highly related to intracranial volume (Buckner et al., 2004).

\section{Results}

\subsection{Demographics and clinical measures}

Demographic and clinical data are presented in Table 1. The ages and sex distributions of the two groups, anxious depression and non-anxious depression, were not significantly different ( $p>0.05$ ). Further, there was no difference in depression severity between the two groups in terms of HAM-D scores $(p>0.05)$ excluding the anxiety/somatization factor scores. There were also no significant differences in the anxiety/somatization factor scores between the two groups $(p>0.05)$. There were significant effects of sex on right FA values $(\mathrm{p}=0.004)$, right amygdalae volume $(\mathrm{p}=0.009)$ and left amygdalae volume $(\mathrm{p}=0.004)$. There were also significant effects 

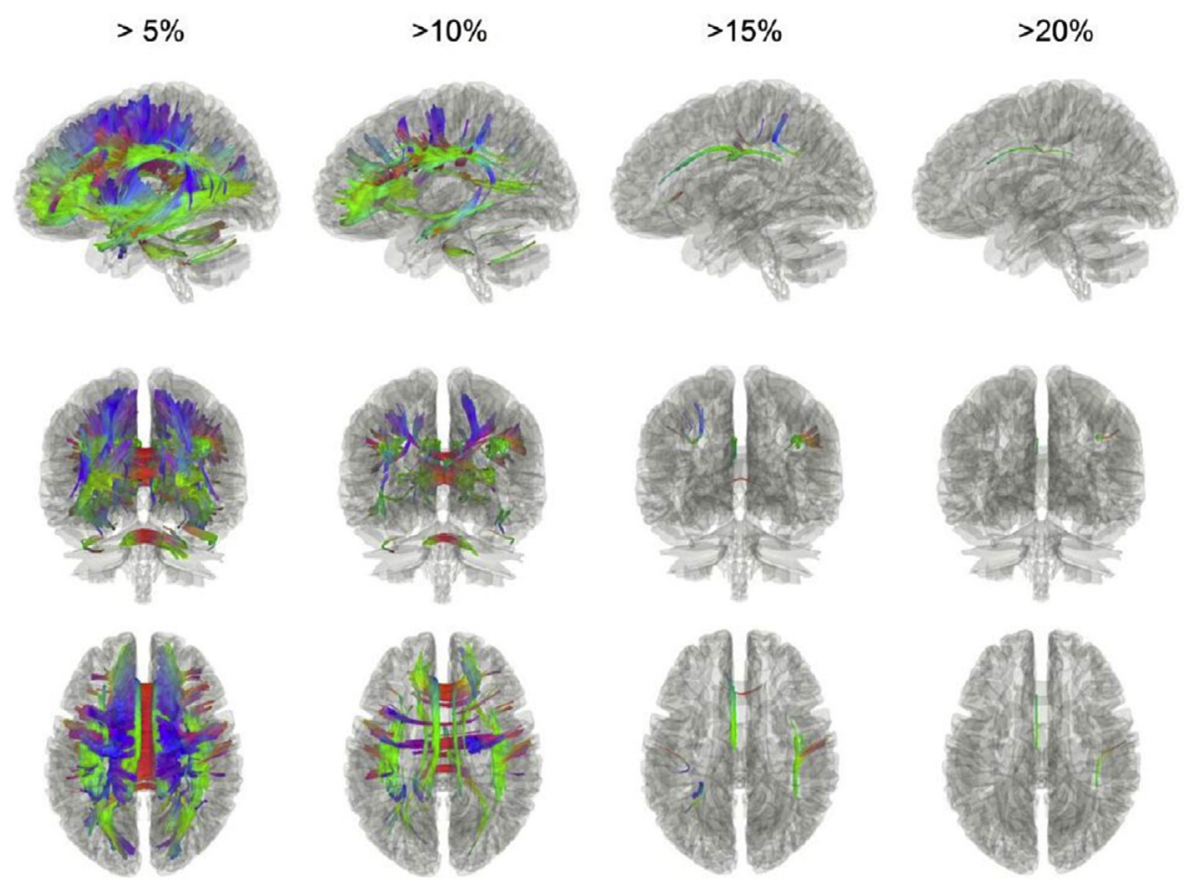

FDR:

.24

.34

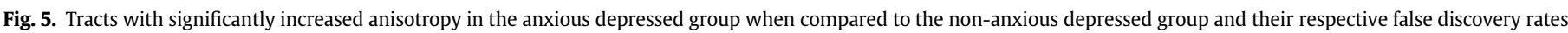

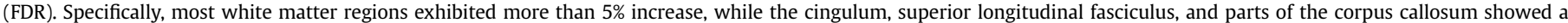
greater increase of more than $10 \%$.

of age on the right and left amygdalae volumes $(\mathrm{p}=0.041)$ and $(\mathrm{p}=0.004)$, respectively.

\subsection{Analysis of group differences with covariates: ANCOVA}

Results of the ANCOVA showed that there was no effect of membership on the FA values or amygdala volumes; Wilks' lambda was not significant, $\mathrm{F}(1,99)=0.74, \mathrm{p}>0.05$ or $\mathrm{F}(1,79)=2.21$, $\mathrm{p}>0.05$, respectively. ANOVAs for each outcome measure are presented in Table 2. As specific phobia is associated with milder levels of clinical distress than other anxiety disorders, we also conducted the analyses excluding the eight individuals with specific phobia. The results were unchanged ( $p>0.05$ ). Effect sizes were small for left FA values $\left(\eta^{2}=0.001\right)$, right FA values $\left(\eta^{2}=0.003\right)$, and left amygdala volumes $\left(\eta^{2}=0.009\right)$. They were slightly larger for right amygdala volumes $\left(\eta^{2}=0.033\right)$. If the study had a larger sample, the differences in right amygdala volume could potentially reach significance.

\subsection{Interaction effects}

Interaction effects were explored, including symptom by group, group by sex, group by age, and site by group. There were no interaction effects (all p values $>0.05$ ).

\subsection{Correlations between clinical measures and neural outcome measures}

Correlations were calculated between clinical measures and neural outcome measures. To avoid conceptual and psychometric overlap, HAM-D anxiety/somatization factor scores were excluded from the HAM-D correlational analysis. There were no significant correlations between DTI-based outcome measures or amygdalae volume and clinical measures at the $p \leq 0.05$ level (Figs. 1-4).

\subsection{Diffusion connectometry results}

The results showed increased anisotropy in the anxious depressed group with percentage of difference from $5 \%$ to $20 \%$ in a number of regions. Nonetheless, the differences have a higher false discovery rates (FDR) around 0.20 (Fig. 5).

\section{Discussion}

Previous studies have shown that white matter health is compromised in both anxiety disorders and depression, so it seems likely that a co-morbid diagnosis could be associated with compounded alterations.

Five studies to date have found reduced FA in the left uncinate fasciculus, the tract connecting the orbitofrontal cortex and the amygdala, of anxious individuals. The depression literature is more mixed, with some studies finding reduced FA in the uncinate fasciculus (Cullen, et al., 2010), but another finding no differences (Olvet, et al., 2016).

In the literature, anxious depression has also been examined through resting state and functional imaging methods. These studies have found decreased connectivity in the medial prefrontal and orbitofrontal cortices (Andreescu et al., 2011), and deficits in amygdalar activations (Etkin and Schatzberg, 2011). Reductions in functional connectivity or activation in brain regions could reflect structural alterations. Existing literature also explores volumetric differences of brain regions in anxious depressed individuals, finding increased gray matter volume in the right superior temporal gyrus compared to non-anxious depressed subjects (Inkster et al., 2011), and reduced volume of the rostral ACC when compared to healthy controls (Van Tol et al., 2010). Decreased volume may suggest reduced structural connectivity in these regions.

The current study sought to explore the discrepancies in the 

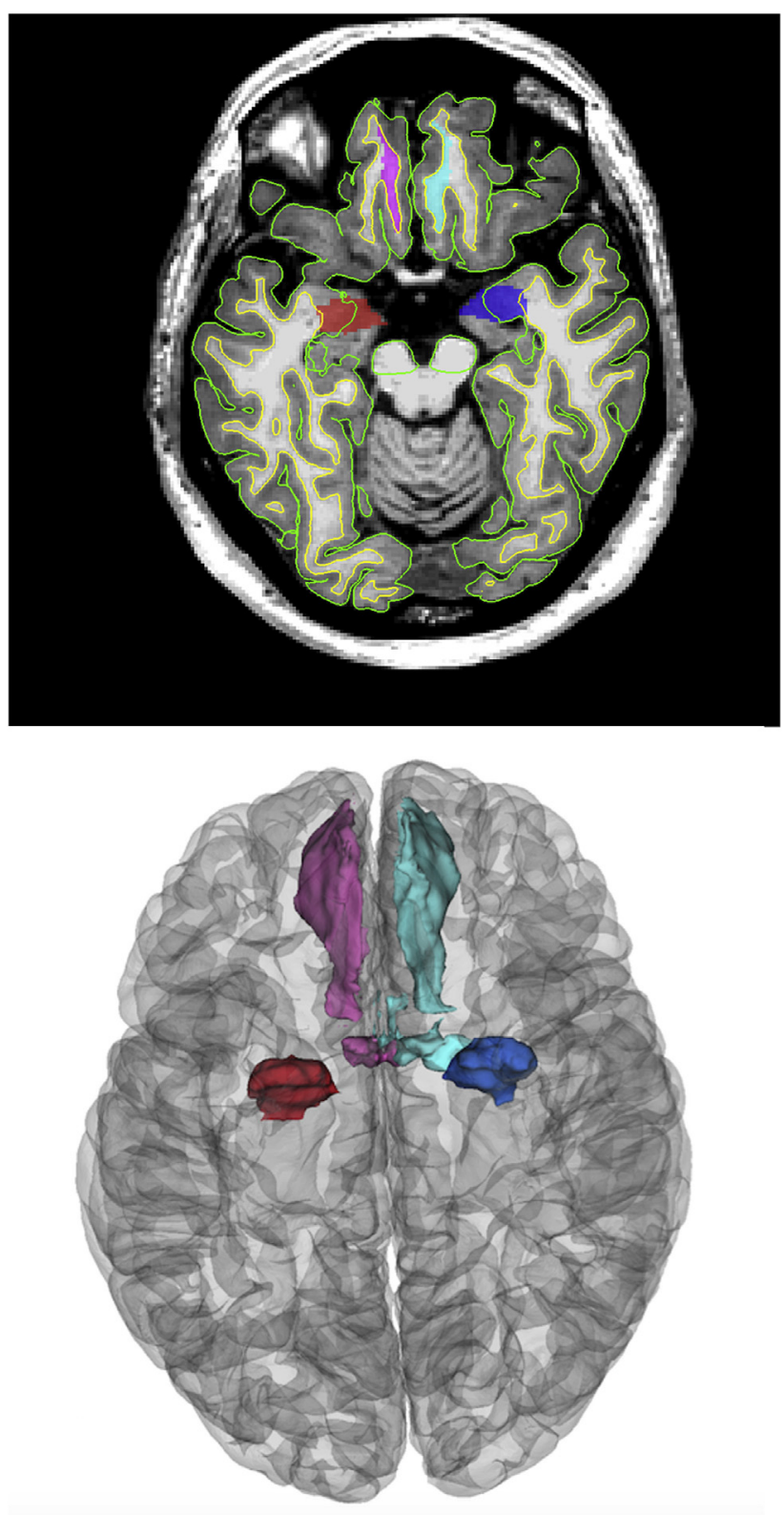

Fig. 6. Example images of the Freesurfer derived manually inspected regions of interest including the bilateral medialorbitofrontal cortices and the amygdalae.

depression literature by considering structural connectivity in depressed subjects with and without co-morbid anxiety disorders. We hypothesized that the effects of anxiety would lead to more severe deficits in the white matter of individuals with anxious depression compared to non-anxious depression. FA was assessed using probabilistic tractography in a priori regions of interest. Spin distribution function was measured using diffusion connectometry. Contrary to our hypothesis, there were no group differences in FA between anxious and non-anxious depressives. Using the whole brain exploratory diffusion connectometry analysis, we observed increased anisotropy in the anxious-depressed group, but these differences were not significant (Fig. 5). It is important to note that the diagnostic and dimensional measures of anxious depression were uncorrelated. This has implications for understanding the previous literature; we cannot assume that results for one measure of anxious depression apply to other measures. However, in this study, using several different and unrelated measures of anxiety and depression, there were still no associations between measures of anxiety and depression and structural connectivity measures.

This study did not replicate past findings of decreased connectivity in more severe psychiatric populations (Cullen, et al., 2010; Taylor, et al., 2007), however, the first group used a smaller adolescent sample $(n=28)$ and the latter group examined a geriatric population. Further, our finding supports our previous study that found no significant associations between white matter FA and depression severity (Olvet, et al., 2016). Existing models of reduced fractional anisotropy in white matter tracts have failed to replicate consistently. The mixed findings in the literature may be a result of differing diagnostic criteria, small sample sizes, medication use, and differences in DTI resolution. Most previous studies do not control for, or examine, co-morbid diagnoses and include individuals taking a wide range of psychotropic medications. In a recent review, most studies show no association between medication use and alteration in DTI outcome measures, however, there are some studies that indicate a significant relationship in multiple brain regions (Hafeman et al., 2012). Furthermore, most of the previous studies include DTI protocols with much fewer diffusion directions and smaller sample sizes. Diffusion weighted acquisitions with $>20$ directions are more likely to accurately quantify the white matter structure and the FA values (Giannelli et al., 2010).

To our knowledge, this is the only reported study to date that examined structural connectivity differences between anxious depression and non-anxious depression. Lastly, it used a DTI sequence that encodes for 64 directions of diffusion and implementation of diffusion connectometry, a whole brain analysis that could be more sensitive to detecting alterations in white matter integrity, providing greater confidence in the result.

The lack of significant differences may also reflect the complexity of both MDD and anxiety disorders. Given the overlap of symptomatology in psychiatric disorders like generalized anxiety disorder and major depressive disorder, it would also be important to examine symptom clusters, rather than diagnosis. It would also be useful to add additional diagnostic groups of anxiety only and healthy controls to determine that there are no interaction effect between anxiety and depression or if there are more global white matter alterations across MDD. As such, the findings of the current paper could be considered preliminary. Another limitation of the current study is the homogeneity in anxiety between the two groups. Lastly, although this sample is moderate in size, we are underpowered to detect smaller effects or interactions. Understanding interaction effects would be important for parsing out whether variables unrelated to clinical status are influencing the relationship between structural connectivity and symptoms/diagnosis. Future studies should use large sample sizes and strive toward improving methods of accurately measuring white matter health. Identifying whether or not there are reliable alterations of structural connectivity in anxious depression would lend a more accurate understanding of the networks and circuitry of psychiatric disorders and could provide a more streamlined and direct treatment for co-morbid disorders.

\section{Acknowledgments}

All Principal Investigators including, Madhukar Trivedi, MD, Patrick McGrath, MD, Myrna Weissman, MD, Ramin Parsey, MD and Maurizio Fava, MD, were involved with protocol design, data 
collection and funding acquisition. All authors contributed to manuscript preparation and approved the final article. This research was supported by a grant from the National Institute of Mental Health (U01-MH092250). The funding source had no involvement in the conduct of research or preparation of this article.

\section{References}

Abhinav, K., Yeh, F.-C., El-Dokla, A., Ferrando, L.M., Chang, Y.-F., Lacomis, D., Friedlander, R.M., Fernandez-Miranda, J.C., 2014. Use of diffusion spectrum imaging in preliminary longitudinal evaluation of amyotrophic lateral sclerosis: development of an imaging biomarker. Front. Hum. Neurosci. 8, 270. http:// dx.doi.org/10.3389/fnhum.2014.00270.

Alexander, D.C., Barker, G.J., 2005. Optimal imaging parameters for fiber-orientation estimation in diffusion MRI. Neuroimage 27 (2), 357-367.

American Psychiatric Association, 2000. Diagnostic and Statistical Manual of Mental Disorders DSM-IV-TR Fourth Edition (Text Revision), fourth ed. American Psychiatric Association, Washington, DC.

American Psychiatric Association, 2013. Diagnostic and Statistical Manual of Mental Disorders, fifth ed. American Psychiatric Association, Washington, DC.

Andreescu, C., Wu, M., Butters, M. a, Figurski, J., Reynolds, C.F., Aizenstein, H.J., 2011. The default mode network in late-life anxious depression. Am. J. Geriatric Psychiatry Official J. Am. Assoc. Geriatric Psychiatry 19 (11), 980-983.

Avenevoli, S., Swendsen, J., He, J.P., Burstein, M., Merikangas, K.R., 2015. Major depression in the national comorbidity survey-adolescent supplement: prevalence, correlates, and treatment. J. Am. Acad. Child Adolesc. Psychiatry 54 (1), 37-44.

Basser, P.J., Mattiello, J., LeBihan, D., 1994. MR diffusion tensor spectroscopy and imaging. Biophysical J. 66 (1), 259-267.

Baur, V., Hänggi, J., Jäncke, L., 2012. Volumetric associations between uncinate fasciculus, amygdala, and trait anxiety. BMC Neurosci. 13, 4.

Behrens, T.E., Berg, H.J., Jbabdi, S., Rushworth, M.F., Woolrich, M.W., 2007. Probabilistic diffusion tractography with multiple fibre orientations: what can we gain? Neuroimage 34 (1), 144-155.

Blackmon, K., Barr, W.B., Carlson, C., Devinsky, O., Dubois, J., Pogash, D., et al., 2011. Structural evidence for involvement of a left amygdala-orbitofrontal network in subclinical anxiety. Psychiatry Res. 194 (3), 296-303.

Buckner, R.L., Head, D., Parker, J., Fotenos, A.F., Marcus, D., Morris, J.C., Snyder, A.Z., 2004. A unified approach for morphometric and functional data analysis in young, old, and demented adults using automated atlas-based head size normalization: reliability and validation against manual measurement of total intracranial volume. Neuroimage 23 (2), 724-738. http://dx.doi.org/10.1016/ j.neuroimage.2004.06.018.

Cullen, K.R., Klimes-Dougan, B., Muetzel, R., Mueller, B.A., Camchong, J., Houri, A., Kurma, S., Lim, K., 2010. Cullen et al., 2010 J. Am. Acad. Child Adolesc. Psychiatry 49, 173-183.

Desikan, R.S., Segonne, F., Fischl, B., Quinn, B.T., Dickerson, B.C., Blacker, D., et al., 2006. An automated labeling system for subdividing the human cerebral cortex on MRI scans into gyral based regions of interest. Neuroimage 31 (3), 968-980.

Etkin, A., Schatzberg, A.F., 2011. Common abnormalities and disorder- specific compensation during implicit regulation of emotional processing in generalized anxiety and major depressive disorders. Am. J. Psychiatry 168, 968-978.

Fava, M., Rush, A.J., Alpert, J.E., Balasubramani, G.K., Wisniewski, S.R., Carmin, C.N., Biggs, M.M., Zisook, S., Leuchter, A., Howland, R., Warden, D., Trivedi, M.H., 2008. Difference in treatment outcome in outpatients with anxious versus nonanxious depression: a STAR*D report. Am. J. Psychiatry 165 (3), 342-351, 21.

Fava, M., Rankin, M.A., Wright, E.C., Alpert, J.E., Nierenberg, A.A., Pava, J., Rosenbaum, J.F., 2000. Anxiety disorders in major depression. Compr. Psychiatry 41 (2), 97-102.

Fava, M., Alpert, J.E., Carmin, C.N., Wisniewski, S.R., Trivedi, M.H., Biggs, M.M., Shores-Wilson, K., Morgan, D., Schwartz, T., Balasubramani, G.K., Rush, A.J. 2004. Clinical correlates and symptom patterns of anxious depression among patients with major depressive disorder in STAR*D. Psychol. Med. 34 (7), 1299-1308, 2004.

First, M., Spitzer, R., Gibbon, M., Williams, J., 1995. Structured Clinical Interview for DSM-IV Axis I Disorders (SCID-I/P, Version 2.0). Biometrics Research Department, New York State Psychiatric Institute, New York.

Fischl, B., Salat, D.H., Busa, E., Albert, M., Dieterich, M., Haselgrove, C., van der Kouwe, A., Killiany, R., Kennedy, D., Klaveness, S., Montillo, A., Makris, N., Rosen, B., Dale, A.M., 2002. Whole brain segmentation: automated labeling of neuroanatomical structures in the human brain. Neuron 33 (3), 341-355.

Giannelli, M., Cosottini, M., Michelassi, M.C., Lazzarotti, G., Belmonte, G., Bartolozzi, C., Lazzeri, M., 2010. Dependence of brain DTI maps of fractional anisotropy and mean diffusivity on the number of diffusion weighting directions. J. Appl. Clin. Med. Phys. 11 (1), 176-190.

Hafeman, D.M., Chang, K.D., Garrett, A.S., Sanders, E.M., Phillips, M.L., 2012. Effects of medication on neuroimaging findings in bipolar disorder: an updated review. Bipolar Disord. 14 (4), 375-410. http://dx.doi.org/10.1111/j.13995618.2012.01023.

Hamilton, M., 1960. A rating scale for depression. J. Neurol. Neurosurg. Psychiatry 23, 56-62.
Hettema, J.M., Kettenmann, B., Ahluwalia, V., McCarthy, C., Kates, W.R., Schmitt, J.E. Silberg, J.L., Neale, M.C., Kendler, K.S., Fatouros, P., 2012. Pilot multimodal twin imaging study of generalized anxiety disorder. Depress. Anxiety 29 (3), 202-209.

Inkster, B., Rao, A.W., Ridler, K., Nichols, T.E., Saemann, P.G., Auer, D.P., Holsboer, F., Tozzi, F., Muglia, P., Merlo-Pich, E., Matthews, P.M., 2011. Structural brain changes in patients with recurrent major depressive disorder presenting with anxiety symptoms. J. Neuroimaging 21, 375-382.

Iscan, Z., Jin, T.B., Kendrick, A., Szeglin, B., Lu, H., Trivedi, M., et al., 2015. Test-retest reliability of freesurfer measurements within and between sites: effects of visual approval process. Hum. Brain Mapp. 36 (9), 3472-3485. http://dx.doi.org/ $10.1002 / \mathrm{hbm} .22856$.

Jbabdi, S., Sotiropoulos, S.N., Haber, S.N., Van Essen, D.C., Behrens, T.E., 2015 Measuring macroscopic brain connections in vivo. Nat. Neurosci. 18, 1546-1555.

Jones, D.K., Basser, P.J., 2004. "Squashing peanuts and smashing pumpkins": how noise distorts diffusion-weighted MR data. Magnetic Reson. Med. 52 (5), 979-993.

Kendler, K.S., Neale, M.C., Kessler, R.C., Heath, A.C., Eaves, L.J., 1992. Major depression and generalized anxiety disorder: same genes, (partly) different environments? Archives General Psychiatry 49 (9), 716-722.

LeDoux, J., 2003. The emotional brain, fear, and the amygdala. Cell. Mol. Neurobiol. 23 (4-5), 727-738.

Linares, I.M., Trzesniak, C., Chagas, M.H., Hallak, J.E., Nardi, A.E., Crippa, J.A., 2012 Neuroimaging in specific phobia disorder: a systematic review of the literature. Rev. Bras. Psiquiatr. 34 (1), 101-111.

Liu, Z., Wang, Y., Gerig, G., Gouttard, S., Tao, R., Fletcher, T., et al., 2010. Quality control of diffusion weighted images. Proc. SPIE Int. Soc. Opt. Eng. 7628 http:// dx.doi.org/10.1117/12.844748.

Olvet, D.M., Delaparte, L., Yeh, F.C., DeLorenzo, C., McGrath, P.J., Weissman, et al., 2016. A comprehensive examination of white matter tracts and connectometry in major depressive disorder. Depress Anxiety 33 (1), 56-65. http://dx.doi.org/ 10.1002/da.22445.

Phan, K.L., Orlichenko, A., Boyd, E., Angstadta, M., Coccarod, E.F., Liberzona, I., Arfanakis, K., 2009. Preliminary evidence of white matter abnormality in the uncinate fasciculus in generalized social anxiety disorder, 2009 Biol. Psychiatry 66, 691-694.

R Core Team, 2013. R: a language and environment for statistical computing. $\mathrm{R}$ Foundation for Statistical Computing, Vienna, Austria. ISBN 3-900051-07-0, URL. http://www.R-project.org/.

Reveley, C., Seth, A.K., Pierpaoli, C., Silva, A.C., Yu, D., Saunders, R.C., Leopold, D.A. Ye, F.Q., 2015. Superficial white matter fiber systems impede detection of longrange cortical connections in diffusion MR tractography. Proc. Natl. Acad. Sci. U. S. A. 112, E2820-E2828.

Rush, A.J., Trivedi, M.H., Ibrahim, H.M., Carmody, T.J., Arnow, B., Klein, D.N. Markowitz, J.C., Ninan, P.T., Kornstein, S., Manber, R., Thase, M.E., Kocsis, J.H., Keller, M.B., 2003. The 16-Item Quick Inventory of Depressive Symptomatology (QIDS), clinician rating (QIDS-C), and self-report (QIDS-SR): a psychometric evaluation in patients with chronic major depression. Biol. Psychiatry 54 (5), 573-583.

Salat, D.H., Greve, D.N., Pacheco, J.L., Quinn, B.T., Helmer, K.G., Buckner, R.L. Fischl, B., 2009. Regional white matter volume differences in nondemented aging and Alzheimer's disease. Neuroimage 44 (4), 1247-1258.

Shin, L.M., Liberzon, I., 2010. The neurocircuitry of fear, stress, and anxiety disorders. Neuropsychopharmacology 35 (1), 169-191.

Stein, D.J., Fineberg, N.A., Bienvenu, O.J., Denys, D., Lochner, C., Nestadt, G., et al., 2010. Should OCD be classified as an anxiety disorder in DSM-V? Depress Anxiety 27 (6), 495-506. http://dx.doi.org/10.1002/da.20699.

Strawn, J.R., Bitter, S.M., Weber, W. a, Chu, W.-J., Whitsel, R.M., Adler, C., et al., 2012 Neurocircuitry of generalized anxiety disorder in adolescents: a pilot functional neuroimaging and functional connectivity study. Depress. Anxiety 29 (11), 939-947.

Steffens, D.C., Taylor, W.D., Denny, K.L., Bergman, S.R., Wang, L., 2011. Structural integrity of the uncinate fasciculus and resting state functional connectivity of the ventral prefrontal cortex in late life depression. PloS One 6 (7), e22697.

Taylor, W.D., MacFall, J.R., Gerig, G., Krishnan, R.R., 2007. Structural integrity of the uncinate fasciculus in geriatric depression: relationship with age of onset. J. Neuropsychiatric Dis. Treat. 3 (5), 669-674.

Trivedi, M.H., McGrath, P.J., Fava, M., Parsey, R.V., Kurian, B.T., Phillips, et al., 2016. Establishing moderators and biosignatures of antidepressant response in clinical care (EMBARC): Rationale and design. J. Psychiatr. Res. 78, 11-23. http:// dx.doi.org/10.1016/j.jpsychires.2016.03.001.

Tromp, D.P.M., Grupe, D.W., Oathes, D.J., McFarlin, D.R., Hernandez, P.J., Kral, T. R. a, et al., 2012. Reduced structural connectivity of a major frontolimbic pathway in generalized anxiety disorder. Archives General Psychiatry 69 (9), 925-934.

Van Tol, M.J., van der Wee, N.J., van denHeuvel, O.A., Nielen, M., Demenescu, L. Aleman, A., Renken, R., Buchem, M., Zitman, F., Veltman, D., 2010. Regional brain volume in depression and anxiety disorders. Archives General Psychiatry 67, 1002-1011.

World Health Organization, 1992. International Statistical Classification of Diseases and Related Health Problems, 10th rev. World Health Organization, Geneva. 1992.

Yeh, F.C., Badre, D., Verstynen, T., 2016. Connectometry: a statistical approach harnessing the analytical potential of the local connectome. Neuroimage 125 , 162-171. http://dx.doi.org/10.1016/j.neuroimage.2015.10.053. 
Yeh, F.C., Tseng, W.Y., 2011. NTU-90: a high angular resolution brain atlas constructed by q-space diffeomorphic reconstruction. Neuroimage 58 (1), 91-99.

Yeh, F.-C., Tang, P.-F., Tseng, W.-Y.I., 2013a. Diffusion MRI connectometry automat ically reveals affected fiber pathways in individuals with chronic stroke. NeuroImage. Clin. 2, 912-921.

Yeh, F.C., Verstynen, T.D., Wang, Y., Fernandez-Miranda, J.C., Tseng, W.Y., 2013b.
Deterministic diffusion fiber tracking improved by quantitative anisotropy. PLoS One 8 (11), e80713.

Zhu, T., Hu, R., Qiu, X., Taylor, M., Tso, Y., Yiannoutsos, C., et al., 2011. Quantification of accuracy and precision of multi-center DTI measurements: a diffusion phantom and human brain study. Neuroimage 56 (3), 1398-1411. http:// dx.doi.org/10.1016/j.neuroimage.2011.02.010. 